\title{
Correction to: Brief Report: Maternal Opioid Prescription from Preconception Through Pregnancy and the Odds of Autism Spectrum Disorder and Autism Features in Children
}

\author{
Eric Rubenstein ${ }^{1}$ (D) Jessica C. Young ${ }^{2} \cdot$ Lisa A. Croen ${ }^{3} \cdot$ Carolyn DiGuiseppi $^{4} \cdot$ Nicole F. Dowling $^{5} \cdot$ Li-Ching Lee $^{6}$. \\ Laura Schieve $^{5} \cdot$ Lisa D. Wiggins ${ }^{5} \cdot$ Julie Daniels $^{2}$
}

Published online: 20 September 2018

(c) Springer Science+Business Media, LLC, part of Springer Nature 2018

\section{Correction to: \\ Journal of Autism and Developmental Disorders https://doi.org/10.1007/s10803-018-3721-8}

The original version of this article unfortunately contained a mistake in Table 2. The "Time of use" should be the column header with the corresponding row with " $\mathrm{N}=126$ " belonging to the "Peri-pregnancy" row. The "Preconception" row should be the row with "N $=17$ ", "Trimester 1" should be the " $\mathrm{N}=29$ " row, "Trimester 2" should be the " $\mathrm{N}=25$ " row, and "Trimester 3" should be the " $\mathrm{N}=47$ " row.

The corrected Table 2 is given below.

The original article can be found online at https://doi.org/10.1007/ s10803-018-3721-8.

Eric Rubenstein

Erubenstein2@wisc.edu

1 Waisman Center, University of Wisconsin-Madison, Rm 529, 1500 Highland Avenue, Madison, WI 53705, USA

2 Department of Epidemiology, Gillings School of Global Public Health, University of North Carolina Chapel Hill, 135 Dauer Dr, Chapel Hill, NC 27599, USA

3 Kaiser Permanente Division of Research, 2000 Broadway, Oakland, CA 94612, USA

4 Department of Epidemiology, Colorado School of Public Health, University of Colorado Anschutz Medical Campus School of Public Health, 13001 E. 17th Place, Aurora, CO 80045, USA

5 National Center on Birth Defects and Developmental Disabilities, Centers for Disease Control and Prevention, 1600 Clifton Road, Atlanta, GA 30329, USA

6 Department of Epidemiology, Johns Hopkins Bloomberg School of Public Health, 615 N. Wolfe Street, Baltimore, MD 21205, USA 
Table 2 Unadjusted and adjusted odds ratios and 95\% confidence intervals for the association between maternal opioid prescriptions in the peri-pregnancy period and child neurodevelopmental outcomes in children born from 2003 to 2012 enrolled in the Study to Explore Early Development

\begin{tabular}{|c|c|c|c|c|c|c|c|c|c|}
\hline \multirow[t]{3}{*}{ Time of use } & \multicolumn{9}{|c|}{ Unadjusted } \\
\hline & \multicolumn{3}{|c|}{$\begin{array}{l}\text { ASD } \\
\mathrm{N}=1369\end{array}$} & \multicolumn{3}{|c|}{$\begin{array}{l}\text { DD-only } \\
\mathrm{N}=938\end{array}$} & \multicolumn{3}{|c|}{$\begin{array}{l}\text { ASD/DD with autism features }{ }^{\mathrm{a}} \\
\mathrm{N}=1845\end{array}$} \\
\hline & $\overline{\mathrm{N}}$ & $\mathrm{OR}^{\mathrm{b}}$ & $95 \% \mathrm{CI}$ & $\overline{\mathrm{N}}$ & OR & $95 \% \mathrm{CI}$ & $\mathrm{N}$ & OR & $95 \% \mathrm{CI}$ \\
\hline Peri-pregnancy ${ }^{\mathrm{c}}$ & 126 & 1.56 & $1.19,2.06$ & 66 & 1.17 & $0.84,1.62$ & 174 & 1.61 & $1.24,2.08$ \\
\hline Preconception $^{\mathrm{d}}$ & 17 & 2.82 & $1.27,6.82$ & 8 & 1.92 & $0.70,5.34$ & 23 & 2.83 & $1.21,6.62$ \\
\hline Trimester 1 & 29 & 1.99 & $1.09,3.63$ & 12 & 1.19 & $0.57,2.50$ & 36 & 1.83 & $1.02,3.26$ \\
\hline Trimester 2 & 25 & 1.52 & $0.84,2.78$ & 21 & 1.88 & $1.00,3.52$ & 38 & 1.72 & $0.99,3.00$ \\
\hline Trimester 3 & 47 & 1.16 & $0.77,1.75$ & 24 & 0.85 & $0.52,1.41$ & 63 & 1.15 & $0.78,1.69$ \\
\hline \multirow[t]{3}{*}{ Time of use } & \multicolumn{9}{|c|}{ Adjusted $^{\mathrm{e}}$} \\
\hline & \multicolumn{3}{|c|}{$\begin{array}{l}\text { ASD } \\
\mathrm{N}=1359\end{array}$} & \multicolumn{3}{|c|}{$\begin{array}{l}\text { DD-Only } \\
\mathrm{N}=927\end{array}$} & \multicolumn{3}{|c|}{$\begin{array}{l}\text { ASD/DD with autism features } \\
\mathrm{N}=1834\end{array}$} \\
\hline & $\mathrm{N}$ & $\mathrm{aOR}$ & $95 \% \mathrm{CI}$ & $\mathrm{N}$ & $\mathrm{aOR}$ & $95 \% \mathrm{CI}$ & $\mathrm{N}$ & $\mathrm{aOR}$ & $95 \% \mathrm{CI}$ \\
\hline Peri-pregnancy & 126 & 1.26 & $0.94,1.68$ & 66 & 1.06 & $0.76,1.49$ & 174 & 1.30 & $0.99,1.71$ \\
\hline Preconception & 17 & 2.43 & $0.99,6.02$ & 8 & 1.94 & $0.68,5.52$ & 23 & 2.64 & $1.10,6.31$ \\
\hline Trimester 1 & 29 & 1.58 & $0.85,2.94$ & 12 & 1.06 & $0.50,2.28$ & 36 & 1.48 & $0.81,2.69$ \\
\hline Trimester 2 & 25 & 1.13 & $0.61,2.12$ & 21 & 1.64 & $0.87,3.12$ & 38 & 1.24 & $0.69,2.22$ \\
\hline Trimester 3 & 47 & 1.02 & $0.66,1.57$ & 24 & 0.85 & $0.52,1.41$ & 63 & 0.96 & $0.64,1.45$ \\
\hline
\end{tabular}

$A S D$ Autism spectrum disorder, $D D$-only developmental delay/disorder without features of ASD, $O R$ odds ratio, $a O R$ adjusted odds ratio, $C I$ confidence interval

${ }^{\text {a }}$ ASD/DD with autism features group includes children with autism spectrum disorder or non-ASD developmental delay/disorders with autism features

${ }^{\mathrm{b}}$ Exposure for case groups were compared to exposure for population controls at each time of use

c 3 months preconception to 1 day prior to childbirth

${ }^{\mathrm{d}} 3$ months preconception to conception

${ }^{\mathrm{e}}$ Adjusted for maternal education, race/ethnicity, smoking during pregnancy, psychiatric condition prior to childbirth, and SEED study period (2003-2006 or 2007-2012). Ns are smaller due to missing covariate data 